\title{
Stereospecific Interactions of Cholesterol in a Model Cell Membrane: Implications for the Membrane Dipole Potential
}

Victoria Oakes ${ }^{1} \&$ Carmen Domene ${ }^{1,2}$

1 Department of Chemistry, 1 South Building, Claverton Down Road, University of Bath, Bath BA2 $7 \mathrm{AY}, \mathrm{UK}$

2 Chemistry Research Laboratory, Mansfield Road, University of Oxford, Oxford, OX1 3TA, UK

Corresponding author: C.Domene@bath.ac.uk

Tel: +44 - (0) 1225386172

Keywords: computer simulations; molecular dynamics; cholesterol; ent-cholesterol; epi-cholesterol; GPCRs

\begin{abstract}
Cholesterol is a major constituent of the plasma membrane in higher-order eukaryote organisms. The effect of cholesterol on the structure and organisation of cell membranes has been studied extensively by both experimental and computational means. In recent years, a wealth of data has been accumulated illustrating how subtle differences in the structure of cholesterol equate to considerable changes in the physical properties of the membrane. The effect of cholesterol stereoisomers, in particular, has been established, identifying a direct link with the activity of specific membrane proteins. In this study, we perform extensive molecular dynamics simulations of phospholipid bilayers containing three isomers of cholesterol, the native form (nat-cholesterol), the enantiomer of the native form (ent-cholesterol), and an epimer of cholesterol that differs by the orientation of the polar hydroxyl group (epi-cholesterol). Based on these simulations, an atomic-level description of the stereospecific cholesterol-phospholipid interactions is provided, establishing a potential mechanism for the perturbation of membrane properties, specifically the membrane dipole potential.
\end{abstract}




\section{INTRODUCTION}

Phospholipid bilayers serve as the fundamental scaffold of all biological membranes, forming a barrier between the cell interior and the surrounding environment. Integral membrane proteins are responsible for the controlled passage of substances across this barrier. Cholesterol is particularly abundant in the plasma membrane of higher-order eukaryotes, and is an important requirement for the functioning of a diverse range of membrane proteins, whilst acting as a crucial regulator of membrane fluidity. ${ }^{1}$ As a result, the specific effect of cholesterol on the structure of a phospholipid bilayer has been under discussion for much of the last century. ${ }^{2}$ Arguably the most striking response to membrane cholesterol is the 'condensing effect', whereby the area per lipid molecule is noticeably lower than would be observed during ideal mixing, and the membrane thickness is increased concordantly, indicating changes in lipid organisation. $^{3}$

In recent years, significant interest has been focused toward characterising the physical effect of naturally occurring cholesterol analogues, as well as synthetic cholesterol isomers and derivatives on lipid membranes. The native form of cholesterol (nat-cholesterol) is an asymmetric amphiphilic molecule, composed of a hydroxyl group attached to a tetracyclic sterane backbone, exhibiting a smooth $\alpha$ face and rough $\beta$ face (Fig. 1A). The enantiomer of nat-cholesterol (ent-cholesterol) is the non-superimposable mirror image (Fig. 1B), exhibiting physiochemical properties indistinguishable to native cholesterol, except for the rotation of plane-polarised light. ${ }^{4-7}$ Subtle changes in cholesterol membrane structure have been shown to exert profound effects on innate physiochemical properties. Certain attributes are known to significantly diverge in membranes containing related cholesterol molecules, including but not limited to epi-cholesterol, a stereoisomer of cholesterol, which differs in the orientation of the hydroxyl group (Fig. 1C), and 7-dehydrocholesterol and desmosterol, cholesterol precursors that differ by a double bond. ${ }^{8-13}$ Membrane dipole potential, the potential difference within the membrane bilayer, exemplifies this phenomenon. A significant increase in dipole potential is observed upon cholesterol replenishment of both model and natural membranes, a lesser increase when 7-dehydrocholesterol and desmosterol are present, whilst replacement with epicholesterol causes the dipole potential to decrease, thus demonstrating that this property is highly dependent on the exact cholesterol structure. ${ }^{14-17}$ The molecular 
mechanism underlying the regulation of membrane dipole potential via cholesterol and related molecules is currently unclear, however.

It has also been demonstrated that variations in cholesterol structure can modulate the functioning of specific membrane proteins, including but not limited to inwardly rectifying potassium channel Kir2.1, ${ }^{18}$ ligand-gated ion channel receptor GABA ${ }_{A}{ }^{19}$ and G-protein coupled receptor 5- $\mathrm{HT}_{1 \mathrm{~A} .}{ }^{20}$ Membrane cholesterol has been suspected to regulate membrane proteins by a direct mechanism, where direct coordination of cholesterol provokes conformational changes in the receptor, or an indirect mechanism, by modifying the membrane properties. Unnatural isomers, ent- and epicholesterol, have proven to be useful experimental probes to determine the exactitude of the interaction of cholesterol with such membrane proteins, and consequently the mechanism of cholesterol action. ${ }^{6,18-21}$ Ent-cholesterol, in particular, is a useful candidate to elucidate specific cholesterol effects, as the membrane properties are unchanged.

To elucidate how cholesterol and related molecules evoke differences in the physical properties of the membrane and the dynamics of membrane proteins, a highresolution description of the specific interactions is required. As a consequence, molecular dynamics (MD) simulations have become an established method to typify the impact of cholesterol on membrane lipids and proteins, in atomic detail. ${ }^{22-24}$ In this study, we have performed all-atom MD simulations of phospholipid bilayers containing 30\% nat-, ent- and epi-cholesterol, as well as a 100\% phospholipid bilayer, in order to determine the atomic behaviour of the cholesterol isomers. The simulations were 500 ns in length, significantly improving the timescale of prior MD simulations examining the dynamics of epi-cholesterol, which were executed for 2 ns. ${ }^{25}$ Key biophysical properties, including area per lipid, cholesterol tilt-angle and solventaccessible-surface area, as well as the identity of crucial cholesterol-phospholipid interactions, were derived from the simulations. The results indicate potential mechanisms by which epi-cholesterol influences the membrane dipole potential and interfere with the functioning of membrane proteins. This is particularly relevant for the functioning of 5- $\mathrm{HT}_{1 \mathrm{~A}}$ receptor, as ligand binding is abated in the presence of the epi-cholesterol. ${ }^{20}$

Figure 1. Chemical structure of (A) nat-cholesterol, (B) ent-cholesterol and (C) epicholesterol molecules, with atom naming used in this study labelled in red. (D) Representative snapshot of a simulation system: a lipid bilayer is solvated in a water box with a final concentration of $150 \mathrm{mM}$ final of $\mathrm{KCl}$. Phosphate atoms of the 
phospholipid molecules, and oxygen atoms of the cholesterol molecules are represented by a brown and red spheres, respectively, to indicate the position of the headgroups. Carbon atoms are shown in cyan. (E) Chemical structure of POPC, with atom naming used in this study labelled in red.
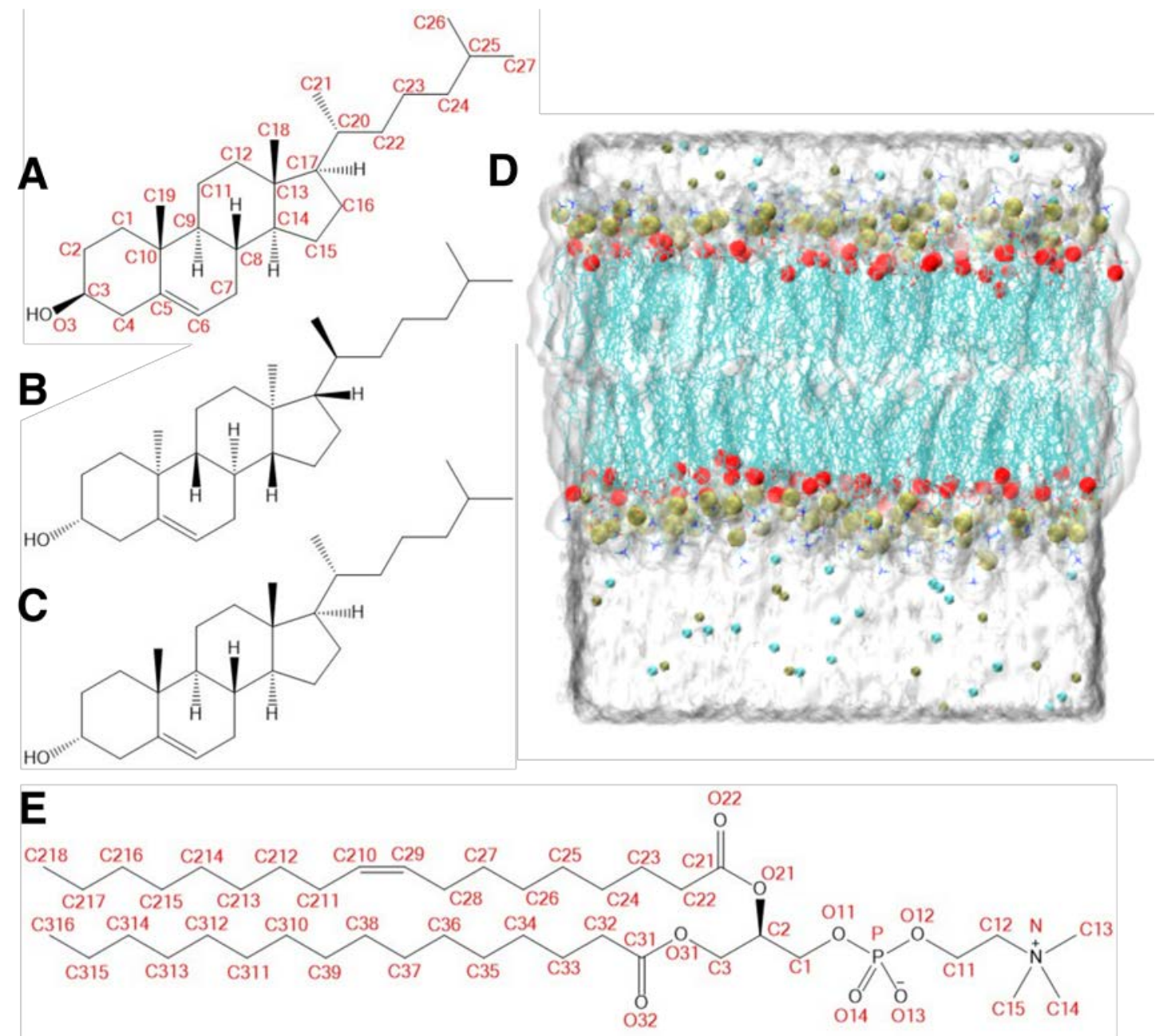

\section{METHODS}

\section{System Setup}

Membranes containing 1- palmitoyl-2-oleoyl-sn-glycero-3-phosphocholine (POPC) molecules and nat-cholesterol molecules were generated using the CHARMM-GUI Membrane Builder. ${ }^{26} 27$ The POPC membrane contained 244 POPC molecules, and the mixed bilayer contained 196 POPC molecules and 84 cholesterol molecules which represents $\sim 30 \%$ cholesterol content to mimic known experimental conditions. The mixed POPC/nat-cholesterol membrane was then modified to generate additional membranes containing the epimer and enantiomer of cholesterol (topology and parameters employed are available upon request). The combined system was then solvated to produce a rectangular water box of dimensions $(92 \times 92 \times 80) \AA^{3} . \mathrm{K}^{+}$and 
$\mathrm{Cl}^{-}$ions were added using the Autoionise Plugin of VMD, to neutralise the system and obtain a $150 \mathrm{mM}$ concentration. ${ }^{29}$ The final systems contained approximately 60,000 atoms, and can be seen in Fig. 1D.

\section{Molecular Dynamics Simulations}

NAMD2.9 was employed to perform molecular dynamics (MD) simulations of the systems throughout. ${ }^{30}$ CHARMM36 parameters were used for phosholipids ${ }^{31}$, with standard parameters for ions ${ }^{32}$ and the TIP3P ${ }^{33}$ model for water. The modified CHARMM36 parameters by Lim et al were used for nat-cholesterol, ent- and epicholesterol molecules. ${ }^{34}$ All systems were equilibrated using the documented CHARMM-GUI protocol. ${ }^{27}$ Unrestrained dynamics for each system was then performed in the NPT ensemble for 500 ns. The Particle Mesh Ewald method was used for the treatment of full-system periodic electrostatic interactions; interactions were evaluated every second timestep with a value of $1 \AA$ to determine grid spacing. ${ }^{35}$ Short-range electrostatic and van der Waals forces were calculated every timestep and up to a cut-off distance of $12 \AA$. A switching distance of $10 \AA$ was chosen to smoothly truncate the non-bonded interactions. Only atoms in a Verlet pair list within a cut-off distance of $13.5 \AA$ were considered, with the list reassigned every 20 steps. ${ }^{36}$ To allow the use of a $2 \mathrm{fs}$ timestep, the SETTLE algorithm was used to make water molecules rigid, and the SHAKE algorithm was used to constrain all other bonds involving hydrogen atoms, utilizing a rigid tolerance of $1 \times 10^{-5} \AA .^{37}$ The Nose-Hoover-Langevin piston was employed to control the pressure with a $200 \mathrm{fs}$ period, $50 \mathrm{fs}$ damping constant and a desired value of 1 atmosphere. ${ }^{38} 39$ The systems were coupled to a Langevin thermostat to sustain a temperature of $298 \mathrm{~K}$ throughout, in line with the experimental conditions used for radiolabel binding assays by Jafurulla et al. ${ }^{20}$

\section{Trajectory Analysis}

The atom names of the cholesterol and POPC molecules referred to in the following text are defined Fig. 1. MEMBPLUGIN was used to perform analyses of (1) membrane thickness, (2) area per lipid, (3) the carbon-deuterium (SCD) order parameter, (4) lipid tilt angle, and (5) lipid interdigitation. ${ }^{40}$ To begin with, the membrane thickness was calculated as the distance between the projected mass distributions of the $\mathrm{P}$ atoms in the phospholipid headgroups in the upper and lower leaflets. To calculate the area per lipid, the simulation box was divided into a Voronoi 
diagram, based on the coordinates of atom O3 in cholesterol molecules and atoms C2, C21 and C31 in POPC molecules (See Fig. 1A and 1D for notation), and the resulting area of the polygons was evaluated, for each bilayer leaflet. The SCD order parameter

was calculated as $S_{C D}=-\frac{1}{2}\left\langle 3 \cos ^{2} \theta-1\right\rangle$, to gauge the motility of the C-H bonds on the phospholipid acyl chains. ${ }^{41}$ The tilt angle was calculated as the angle between the vector defined by the C10 and C13 cholesterol atoms and the bilayer normal, which is aligned to the z-axis throughout the simulation trajectories. The degree of acyl chain interdigitation was measured via a coordination-based fraction, obtained by evaluating the number of heavy atoms within $4 \AA$ (default value) of atoms in the opposing leaflets. ${ }^{40}$ Density profiles were calculated using the Density Profile Tool of VMD. ${ }^{42}$ The solvent-accessible surface area and radial distribution functions were also calculated using built-in VMD tools. Further analysis was performed using inhouse TCL scripts. A $4 \AA$ distance cut-off was used for the calculation of intermolecular charge pairs and atomic interaction pairs between heavy atoms. A 3.5 $\AA$ distance and $35^{\circ}$ angle cut-off was used for the calculation of H-bonding. The lifetime of epi-cholesterol dimers was calculated by considering the period between $\mathrm{H}$-bond formation and disruption of the H-bond for a minimum of $0.5 \mathrm{~ns}$.

Reformation after this interval is considered as a new dimer, and hence lifetime. The initial 200 ns of the simulation trajectories were considered as an equilibration period, following assessment of the fluctuations of the membrane thickness and area per lipid (Table S1). These properties were regarded as converged by $500 \mathrm{~ns}$, thus all analysis is performed on the 200-500 ns time interval. Electrostatic potential of the simulation system was calculated using the "PMEpot” plugin of VMD, following the methodology of Aksimentiev and Schulten. ${ }^{43}$

\section{RESULTS}

In the first instance, global membrane properties have been calculated to elucidate the differences detectable using the MD simulation protocol described in this study, and the conformance of such results with known experimental data. In the POPC simulation, the membrane thickness and the area per lipid (Fig. S1) are calculated as $40 \pm 1 \AA$ and $63 \pm 1 \AA^{3}$ respectively (Table 1 ), in close agreement with accepted experimental values. ${ }^{44}$ The addition of cholesterol causes a net reduction of total bilayer area, with the overall average area per lipid in the system reducing to $45 \pm 1$ $\AA^{3}$ in CHL and $46 \pm 1 \AA^{3}$ in ENT, characteristic of the well-known condensing effect 
of cholesterol. ${ }^{3}$ This effect is slightly offset in EPI, with an overall average area per lipid of $48 \pm 1 \AA^{3}$, primarily as a result of an increased area of POPC molecules (Table 1). Correspondingly, an increase in membrane thickness is observed in CHL (46 $\pm 1 \AA)$, ENT (46 $\pm 1 \AA$ ) and to a lesser degree in EPI (45 $\pm 1 \AA$ ). The extent to which the components of the upper and lower leaflets overlap, known as lipid interdigitation, are expectantly inversely correlated, displaying an increase in interdigitation alongside a decrease in thickness (Table 1). 
Table 1. Summary of key membrane physical properties ( \pm SD) obtained from MD simulations on the 200-500 ns time interval.

\begin{tabular}{|l|l|c|l|l|l|}
\hline \multicolumn{2}{|c|}{} & POPC & CHL & ENT & EPI \\
\hline \multirow{3}{*}{ Thickness $(\AA)$} & POPC & $39.8 \pm 0.9$ & $46.3 \pm 0.9$ & $45.9 \pm 0.9$ & $44.8 \pm 1.0$ \\
\cline { 2 - 6 } & Sterol & - & $35.7 \pm 0.8$ & $35.6 \pm 0.7$ & $33.2 \pm 0.8$ \\
\hline \multirow{2}{*}{$\begin{array}{l}\text { Area per Lipid }\left(\AA^{3}\right) \\
\text { Tilt Angle }\left({ }^{\circ}\right)\end{array}$} & POPC+Sterol & $62.5 \pm 1.2$ & $45.4 \pm 0.7$ & $45.9 \pm 0.7$ & $47.4 \pm 0.9$ \\
\cline { 2 - 6 } & POPC & $62.5 \pm 0.9$ & $53.3 \pm 0.7$ & $53.9 \pm 0.6$ & $55.7 \pm 0.8$ \\
\cline { 2 - 6 } & Sterol & - & $27.2 \pm 0.8$ & $27.0 \pm 0.8$ & $28.1 \pm 1.1$ \\
\hline Sterol & - & $14.4 \pm 1.2$ & $15.3 \pm 1.2$ & $19.0 \pm 1.5$ \\
\hline Interdigitation (\%) & & $5.6 \pm 0.4$ & $3.4 \pm 0.2$ & $3.5 \pm 0.2$ & $3.8 \pm 0.2$ \\
\hline
\end{tabular}

Figure 2. (A) Distribution of cholesterol tilt angles, normalized to 1 with a bin width of 1. SCD order parameter of (B) 16:0 saturated acyl chain at the glycerol sn1 position (C3) and (C) 18:1 unsaturated acyl chain at the glycerol sn2 position (C2). Mass density profiles of cholesterol (D) C3 and (E) O3 atoms, in the lower (left) and upper (right) leaflets, following alignment of the POPC phosphate atoms, normalized to 1 with a bin width of 0.5. SASA per (F) POPC and (G) cholesterol molecule, normalized to 1 with a bin width of 10 and 1 respectively. CHL, ENT, EPI and POPC simulations are displayed in red, blue, green and black respectively. 

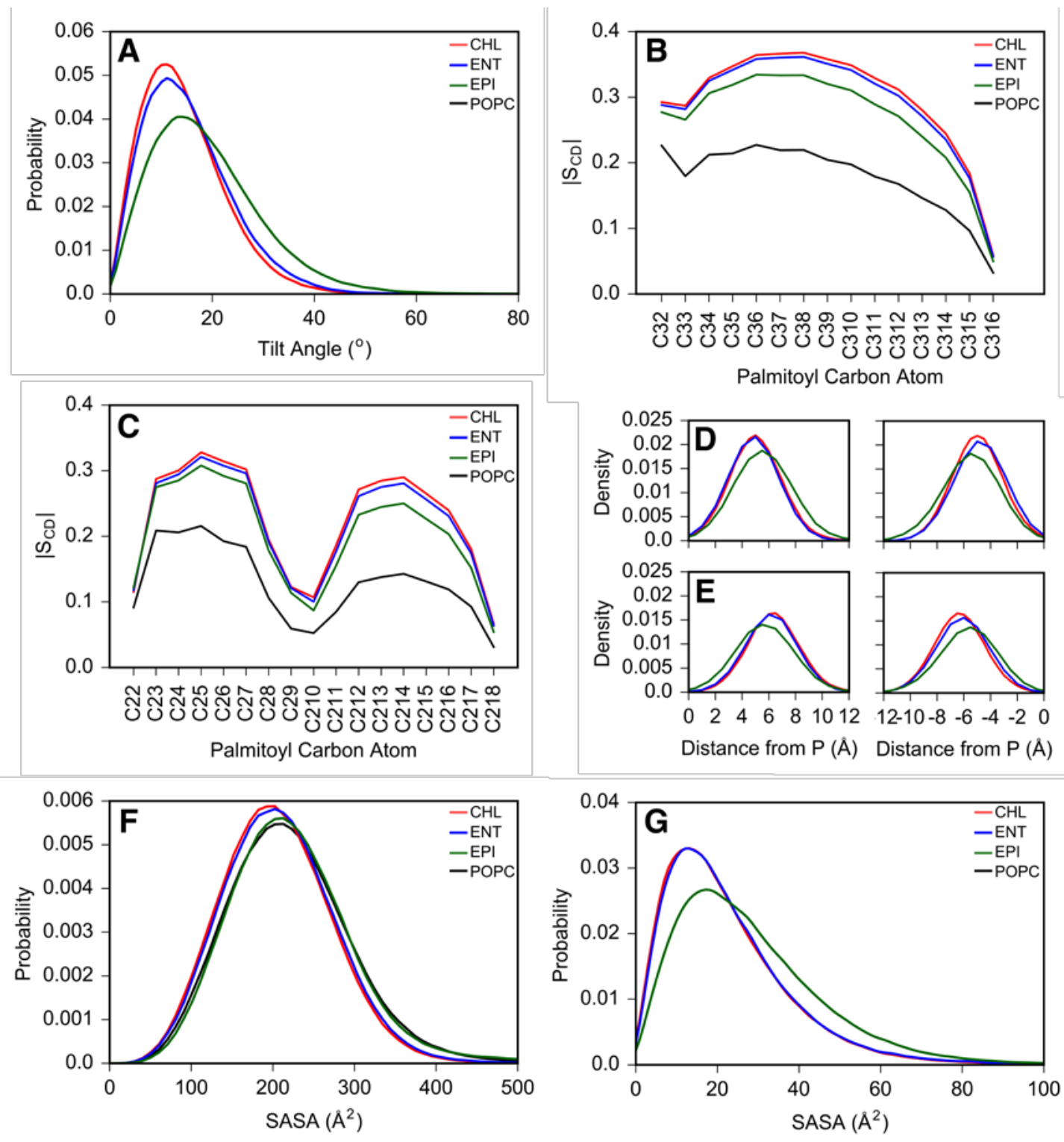

Such differences in the membrane physical properties arise from differences in the position and orientation of cholesterol in the bilayer, and the synergistic interaction with other bilayer components. ${ }^{45}$ Cholesterol tilt angle is an important quantity, which is closely related to the ability of cholesterol to order its environment. ${ }^{46}$ In this example, CHL and ENT display the narrowest distribution of tilt angles, with mean values of $14 \pm 1^{\circ}$ and $15 \pm 1^{\circ}$, respectively, representing a conformation almost parallel to the bilayer normal (Fig. 2A). The distribution of tilt angles in EPI is noticeably more spread, with an increased mean of $19 \pm 2{ }^{\circ}$. This effect is reflected in the calculated $S_{C D}$ order parameter, which is commonly used as a measure of lipid tail organisation, and can be directly compared to experimentation. ${ }^{47-49}$ Overall, $\mathrm{S}_{\mathrm{CD}}$ values increase in the cholesterol-containing bilayers, relative to POPC alone (Fig. 2B-C), indicative of the ordering effect of cholesterol that is well established in the 
literature. ${ }^{50}$ This effect has been attributed to lipid packing around the rigid cholesterol frame. EPI exerts a weaker ordering effect overall, relative to CHL and ENT, with the greatest differences observed in the saturated regions of the acyl chains. These results contribute to a wealth of previous literature, which note the specificity of membrane ordering with regards the atomic structure of cholesterol. ${ }^{51}$ To directly compare the position of the cholesterol hydrophobic ring system and the hydrophilic hydroxyl group in the membrane, the mass density profiles of the C3 and O3 atoms (defined in Fig. 1A), following alignment of PC phosphate atoms, can be calculated (Fig. 2D-E). The C3-O3 maxima of EPI overlap at $~ 5.5 \AA$ resulting from the axial conformation of the hydroxyl group, suggesting that whilst C3 adopts a shallower position in the membrane ( $\sim \AA \AA$ in CHL and ENT) whilst O3 is more buried ( $\sim 5 \AA$ in CHL and ENT).

Figure 3. (A-B) Average interaction frequency between cholesterol and POPC atoms (specified). Interactions are calculated using a $4 \AA$ criterion between heavy atoms.
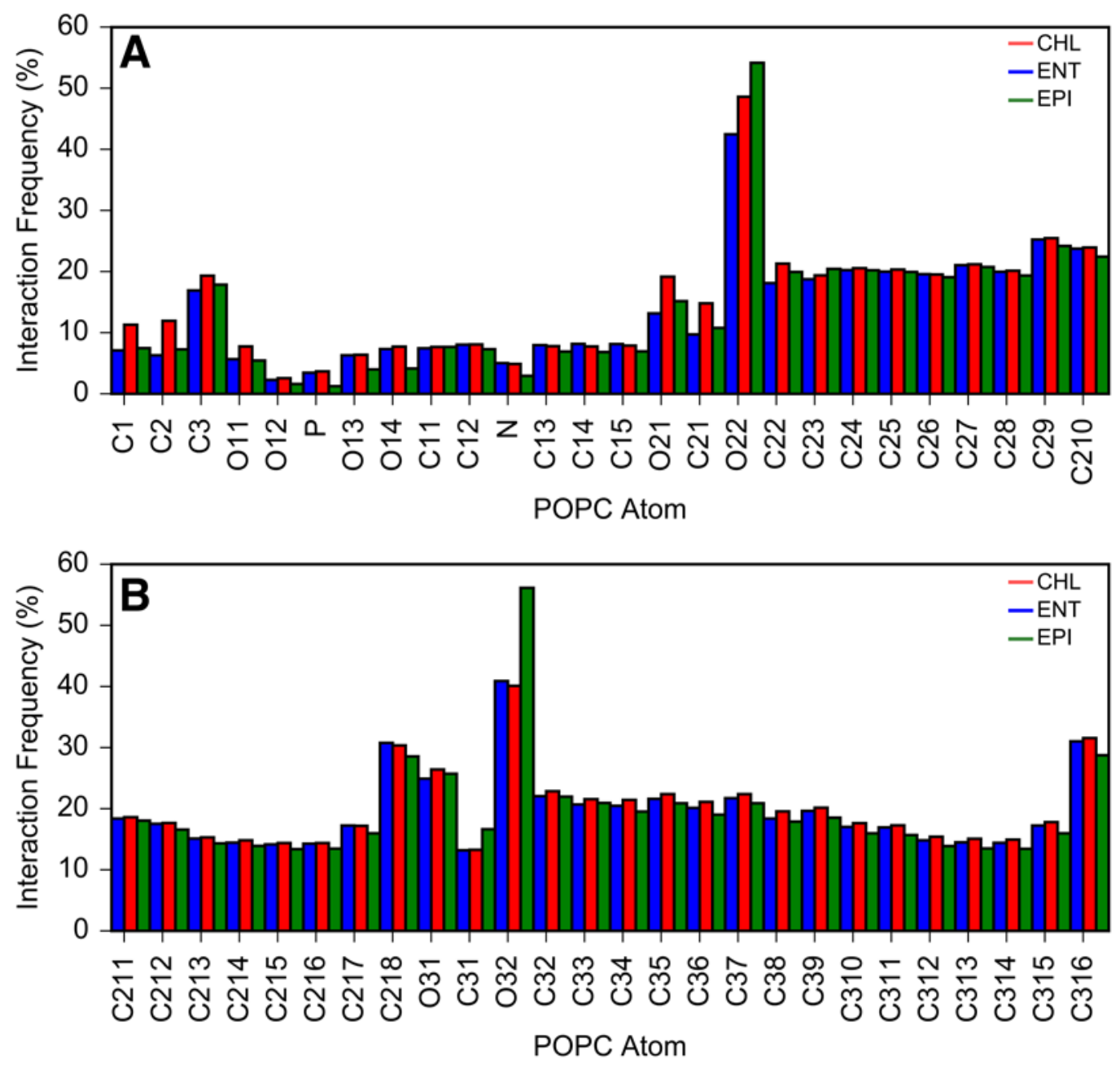
To assess how this altered position affects the organisation of the membrane on an atomic-level, the average interaction frequency between individual POPC and cholesterol atoms has been calculated (Fig. 3). In general, headgroup atoms exhibit an average interaction frequency of $\sim 0-10 \%$ of POPC, whilst tail atoms and those in the linker region, display average interaction frequencies in the $10-30 \%$ range, with EPI consistently presenting lower values. The carbonyl groups of the ester linkages (O22 and O32) are the exceptions to this, with average interaction frequencies in excess of $40 \%$ and elevated average interaction frequencies in EPI (54\%, 56\%) are relative to CHL (42\%, 41\%) and ENT (49\%, 40\%). The observed net increases can be attributed to enhanced interactions with C1, C5, C6 and C7 atoms, offsetting the reduced contact interface with $\mathrm{O} 3$.

To assess the impact of such differences at the membrane-water interface, the solvent accessible surface area (SASA) per molecule has been calculated for POPC and each sterol species (Fig. 2F-G and Table 2). The SASA of POPC in a single component membrane is calculated as $\sim 220 \AA^{3}$, which reduces by $\sim 15 \AA^{3}$ in CHL, $12 \AA^{3}$ in ENT and remarkably increases by $\sim 5 \AA^{3}$ in EPI, despite a decrease in the area per lipid for all cholesterol isomers relative to POPC-only. These observations provide evidence that SASA, and hence POPC-solvent interactions, are modulated by specific lipidcholesterol interactions. It is interesting to note that the hydration of POPC headgroups decreases by 4\% in CHL and ENT, and 2\% in EPI, as calculated from the individual RDFs of all POPC atoms (Fig. S2).

Table 2. Average SASA ( \pm SD) of membrane lipids (the standard error of reported values is less than 0.1)

\begin{tabular}{|l|l|l|l|l|}
\hline SASA $\left(^{\AA^{2}}\right)$ & POPC & CHL & ENT & EPI \\
\hline POPC & $220 \pm 75$ & $205 \pm 67$ & $208 \pm 68$ & $225 \pm 81$ \\
\hline Sterol & - & $22 \pm 16$ & $23 \pm 16$ & $28 \pm 18$ \\
\hline
\end{tabular}

With regard to the SASA of cholesterol, CHL $\left(22 \AA^{3}\right)$ and ENT (23 $\left.\AA^{3}\right)$ display similar values, increasing to $28 \AA^{3}$ in EPI (Table 2). This increase can be attributed to numerous effects, such as the increased area per cholesterol and tilt angle, the elevated position in the membrane and exposure of ring atoms as a result of the orientation of the hydroxyl group. The net increase in hydration in EPI is $~ 10 \%$, relative to CHL and ENT, as calculated from the individual RDFs of all cholesterol atoms (Fig. S3). It is worth noting that a decrease is observed for O3, signalling that 
interactions with hydrophobic atoms are solely responsible for the observed increase. This will likely perturb the electrostatics of the cholesterol-water interfacial region. H-bonding is a further important feature of the membrane-water interface. Approximately, all CHL and ENT molecules participate in at least one H-bond, via the cholesterol hydroxyl group, with $42 \%$ of molecules associated with PC headgroups and $60 \%$ of molecules H-bonding with water, in both cases. In contrast, $70 \%$ of EPI molecules are involved in H-bonding, as a result of diminished interactions with both PC headgroups (25\%) and solvent (37\%), and the evolution of cholesterol homo-dimers (8\%). Dissection into the individual components of the PC headgroup (Fig. 4A, see Fig. 1D for atom names), identifies the largest reduction of H-bonding with atoms $\mathrm{O} 13$ (-3\%) and $\mathrm{O} 14$ (-3\%) in the phosphate group, and O22 ($6 \%)$ at the apex of the unsaturated acyl chain at the sn2 acyl position, whilst $\mathrm{H}$ bonding with $\mathrm{O} 32$ on the saturated acyl chain at the sn1 position is generally conserved. The remaining reductions can be attributed to atoms C12, C13, C14, C15, O11 and O12. The total number of interactions with the cholesterol hydroxyl group displays an analogous pattern.

With regards to the hydration of the PC headgroups, approximately $18 \%$ of POPC molecules H-bond with CHL and ENT, whilst 10\% H-bond with EPI. The reduction in PC-sterol H-bonds in EPI is overcompensated for by an increase in solvent $\mathrm{H}$ bonds, resulting in a total of 9.4, 9.4 and 9.5 H-bonds per POPC molecule, in the CHL, ENT and EPI simulations respectively. Thus it is effectively unchanged by the presence of different cholesterol isomers. This is noticeably less than in the pure POPC membrane simulation ( 10.3 H-bonds per POPC molecules), which can largely be attributed to elevated H-bonding of phosphate oxygen atoms (O13 and O14; 32\%), choline methyl groups (C13, C14, C15; -21\%) and carbonyl atoms within the ester linkages (O22, O32; -20\%) in the absence of cholesterol (Fig. 4B).

Figure 4. (A) Frequency of PC-sterol H-bonds, per cholesterol molecule. (B) Frequency of PC-water H-bonds, per POPC molecule. CHL, ENT, EPI and POPC simulations are displayed in red, blue, green and black respectively. (C) Licorice representation of a POPC molecule coloured according to the percentage interaction with the cholesterol hydroxyl group. A distance criterion of $4 \AA$ between heavy atoms is used in this case. 

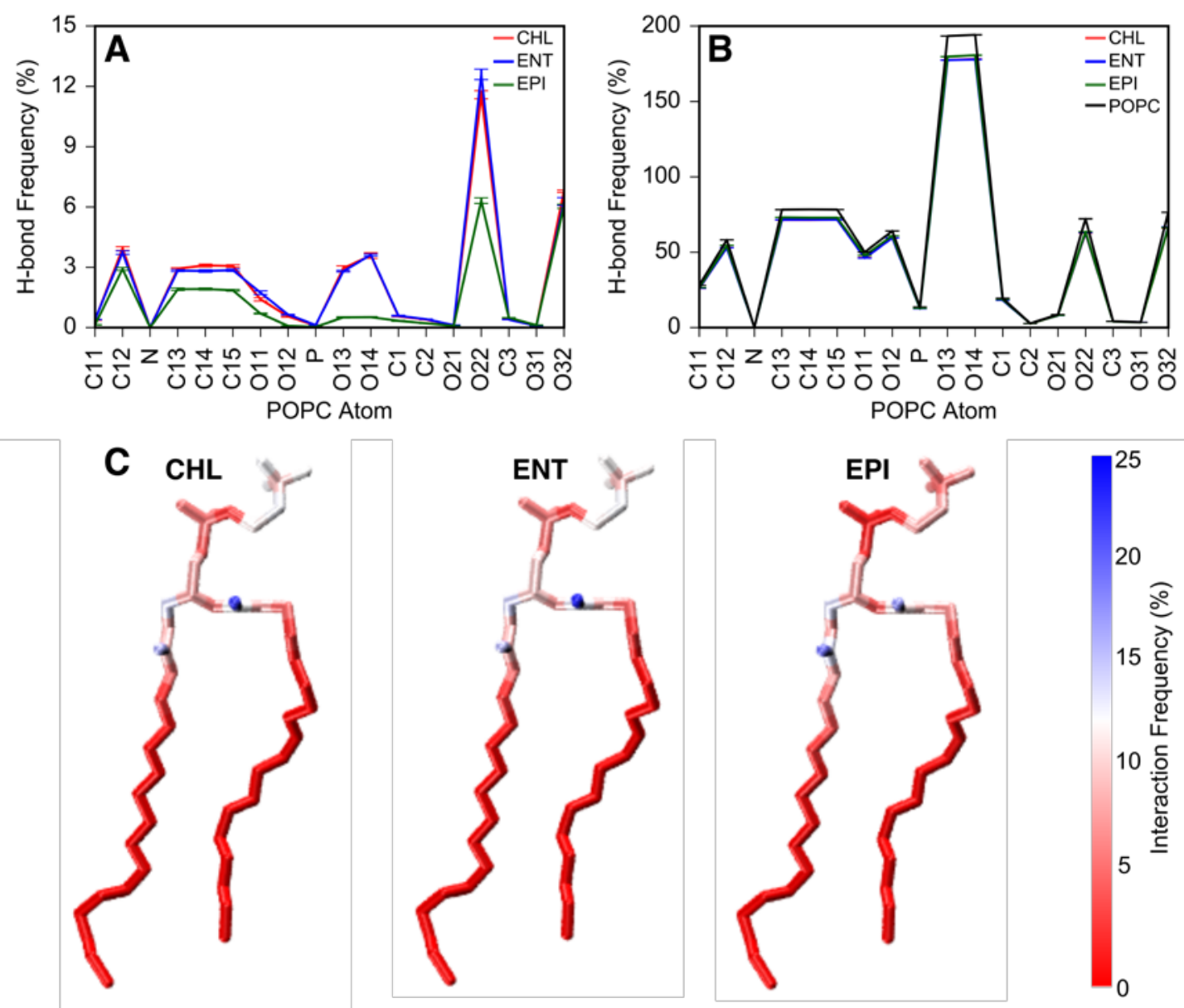

Charge-pairs also contribute significantly to the dynamics of the membrane headgroup region. Intermolecular charge-pairs can be formed between the methyl groups of the positively charged choline moiety of PC, and negatively charged groups in cholesterol and PC headgroups, including the cholesterol hydroxyl (O3), the nonester phosphate oxygen atoms $(\mathrm{O} 13, \mathrm{O} 14)$ and the carbonyl atoms within the ester linkages at the apex of the acyl chains (O22, O32). The frequency of CHL-PC charge pairs is significantly perturbed in EPI, likely as a direct result of the orientation of the O3 hydroxyl (Fig. 5A). Relative to the POPC-only membrane, the number of PC-PC charge-pairs is increased by approximately 15\%, 14\% and 3\%, in CHL, ENT and EPI respectively. The number of charge-pairs involving $\mathrm{O} 13$ and $\mathrm{O} 14$ (POPC < EPI < $\mathrm{CHL} \approx \mathrm{ENT}$ ) is inversely correlated in the observed trend for the values calculated for the area per lipid (POPC $>$ EPI $>$ CHL $\approx$ ENT) (Fig. 5B). It is apparent, however, that the distance between PC headgroups does not dictate the frequency of choline chargepairs with $\mathrm{O} 22$ and $\mathrm{O} 32$ (Fig. 5C-D), and instead is likely directly influenced by the cholesterol headgroup. As a result, the number of charge-pairs for $\mathrm{O} 22$ displays the trend $\mathrm{EPI} \approx \mathrm{POPC}<\mathrm{CHL} \approx \mathrm{ENT}$, whilst the trend $\mathrm{EPI}<\mathrm{POPC} \approx \mathrm{CHL} \approx \mathrm{ENT}$ is 
observed for O32. Thus, the increased frequency of O13/O14 choline charge-pairs in EPI, relative to POPC, is offset by the reduction in $\mathrm{O} 32$ choline charge-pairs, resulting in an overall pattern of $\mathrm{EPI} \approx \mathrm{POPC}<\mathrm{CHL} \approx \mathrm{ENT}$.

Figure 5. Radial distribution function of (A) cholesterol O3, (B) lipid O13/O14, (C) lipid O32, and (D) O22 atoms with C12, C13 and C14 atoms of the lipid headgroup choline region, to represent the distribution of intermolecular charge pairs. CHL, ENT, EPI and POPC simulations are displayed in red, blue, green and black respectively.
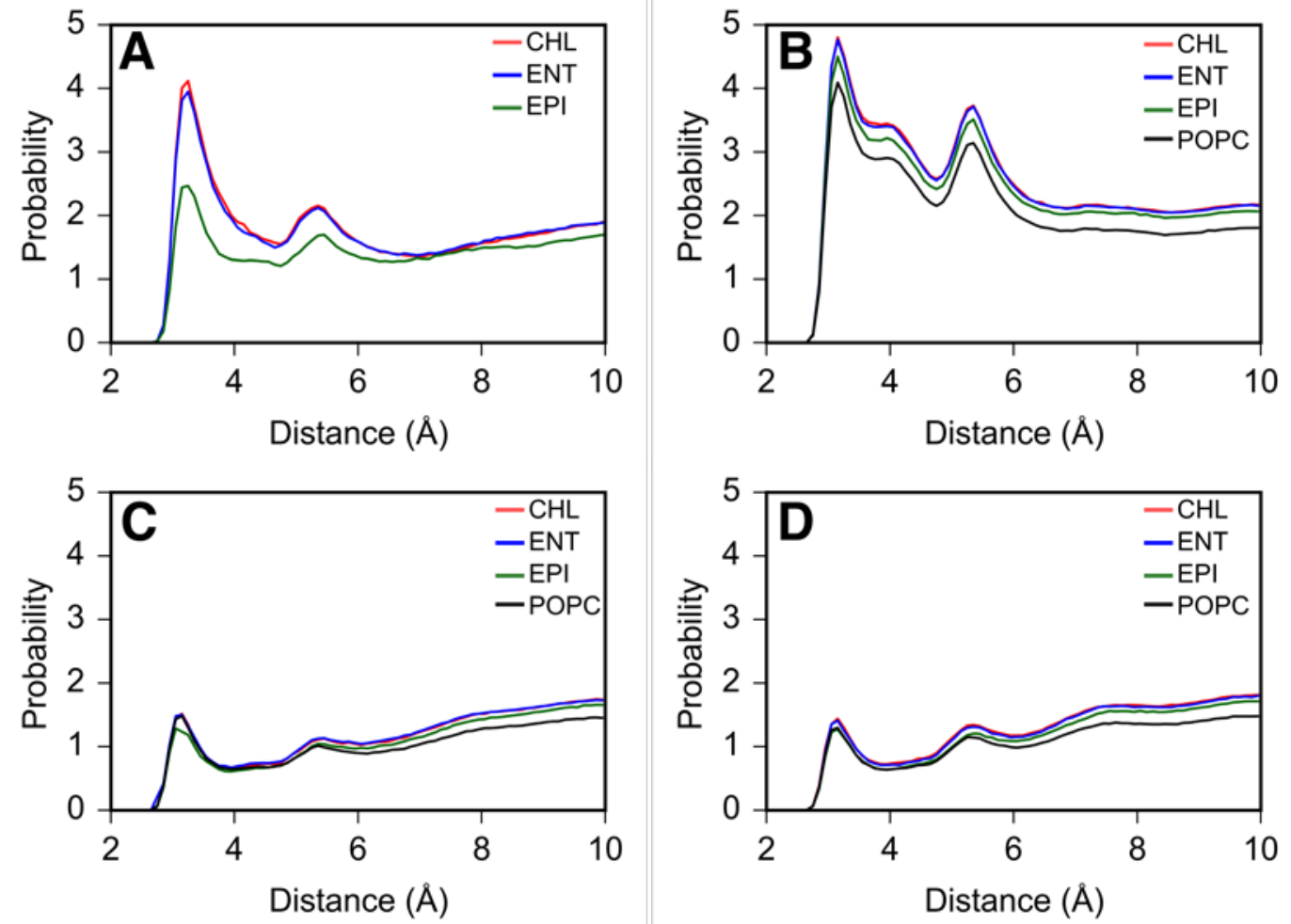

It is conceivable that a correlation exists between the formation of charge pairs between the choline methyl groups and the carbonyl oxygen atoms involved in the ester linkages, and the $\mathrm{P}^{+}-\mathrm{N}^{-}$dipole of the phospholipid headgroups. The average $\mathrm{P}^{+}$$\mathrm{N}^{-}$vector relative to the bilayer normal is calculated as $68 \pm 27^{\circ}$ throughout, however, indicating this is not the case (Fig. 6A). Instead, it is apparent the charge density on the bilayer surface is significantly perturbed, demonstrated by the RDF of $\mathrm{K}^{+}$and $\mathrm{Cl}^{-}$ ions with respect to phospholipid and cholesterol molecules (Fig. 6B and C, respectively). A maximum is observed at $\sim 2.65 \AA$ for $\mathrm{K}^{+}$ions throughout, with the probability at this point following the trend: POPC (0.54) > ENT (0.45) > CHL (0.41) $>$ EPI (0.17). In the case of $\mathrm{Cl}^{-}$, a unique peak is observed at $2.65(0.05)$ which is absent elsewhere. The reduced density of cations, and concurrently, increased density of anions on the bilayer surface reveals the elevated positive potential of the EPI bilayer surface, relative to CHL and ENT. This can also be illustrated by the 
electrostatic potential of the cholesterol isomers; considering the bilayer centre as 0 $\mathrm{mV}, \mathrm{CHL}$, ENT and EPI display values of $-226 \mathrm{mV},-220 \mathrm{mV}$ and $-162 \mathrm{mV}$, respectively (Fig. 6D). The electrostatic potential of the complete CHL, ENT and EPI simulation systems vary to a lesser extent, with calculated values of $-801 \mathrm{mV},-807$ $\mathrm{mV}$ and $-783 \mathrm{mV}$.

Figure 6. (A) Angle between $\mathrm{P}^{+}-\mathrm{N}^{-}$vector relative to the bilayer normal. Radial distribution function between (B) $\mathrm{K}^{+}$and (C) $\mathrm{Cl}^{-}$ions and phospholipid and cholesterol molecules. Electrostatic potential of (D) cholesterol molecules and (E) the full simulation system, relative to the bilayer center. CHL, ENT, EPI and POPC simulations are displayed in red, blue, green and black respectively.
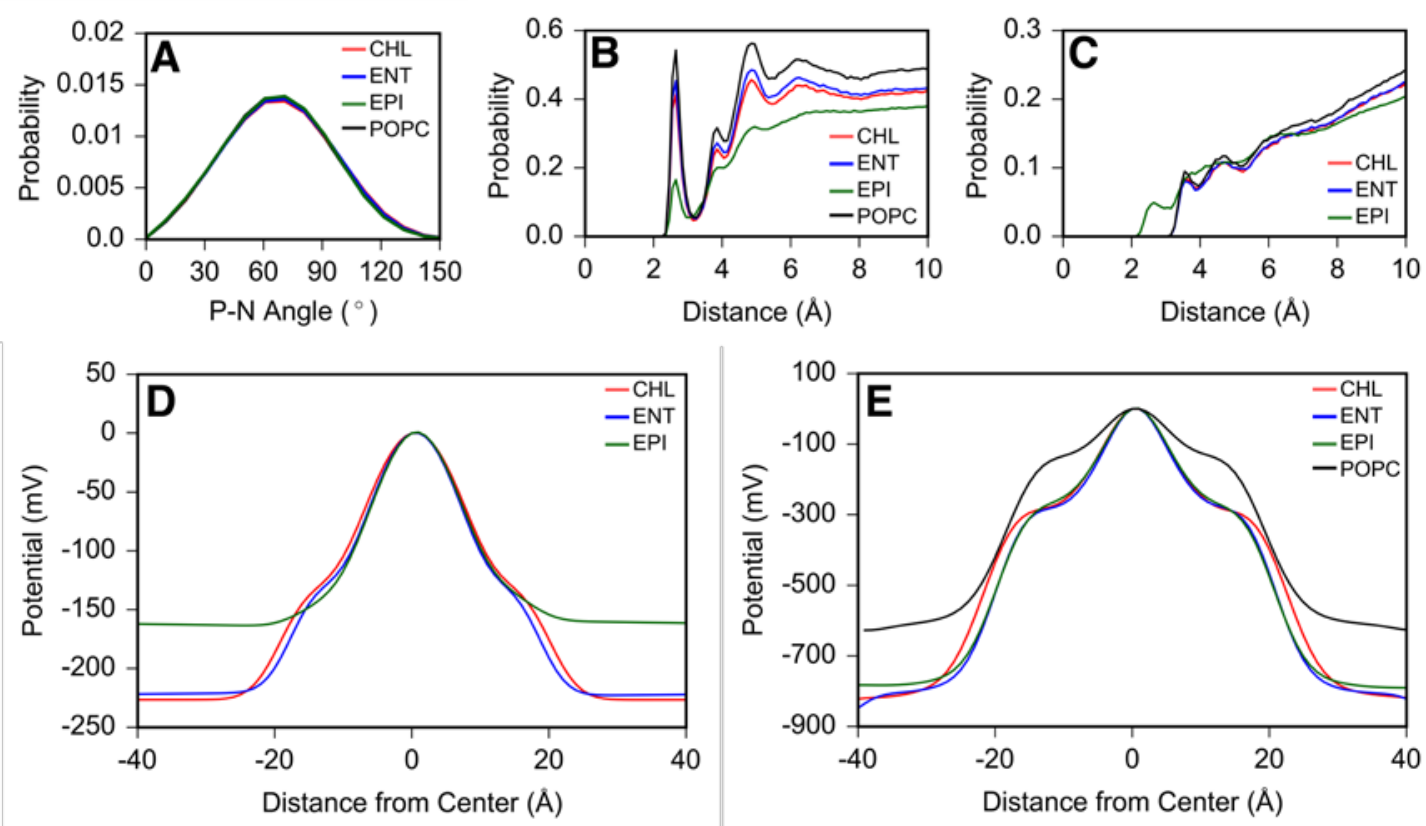

The nature of intermolecular cholesterol interactions is also worth considering. The calculated RDF of the cholesterol C3 atoms, up to a distance of $10 \AA$, is shown in Fig. 7A. According to this data, all cholesterol isomers are guaranteed to be within $\sim 6.5 \AA$ of another cholesterol molecule, yet the characteristics of cholesterol self-association are considerably different in EPI. The first RDF peak in CHL and ENT lies at $4 \AA$, whereas in EPI the first RDF peak $\sim 5 \AA$ and is approximately twice as probable, suggesting the alternative orientation of the hydroxyl group simultaneously acts as a spacer and a stabiliser. It should be noted that the concentration and initial configuration of cholesterol isomers is identical between simulations, and thus differences can be predominantly attributed to this feature. These results are consistent with the noticeable population of intermolecular cholesterol H-bonds identified in EPI by prior H-bond analysis, calling attention to the evolution of novel cholesterol dimers in this case. Using H-bond frequency as an indicator of dimer 
formation, the average lifetime of epi-cholesterol dimers has been calculated as $0.9 \pm$ $1.1 \mathrm{~ns}$, with a maximum lifetime of $\sim 11$ ns (Fig. 7B). Characterisation of the interfacial region between two bound molecules (Fig. 7C) reveals the crucial interactions are localised in the hydroxyl region, with the oxygen interacting with $\sim 4$ atoms on the adjacent molecule simultaneously; the most probable interactions in descending order are O3, C3, C6 and C1 (Fig. 7D).

Figure 7. (A) RDF of cholesterol C3 atoms. CHL, ENT and EPI systems are shown in red, blue and black respectively. (B) Weighted histogram of dimer lifetime, calculated as the continuous occurrence of inter-cholesterol H-bonds. (C) Average number of interaction pairs between epi-cholesterol molecules exclusively involved in dimers (D) Interaction frequency between a hydroxyl $\mathrm{O} 3$ and an adjacent epi-cholesterol molecule.
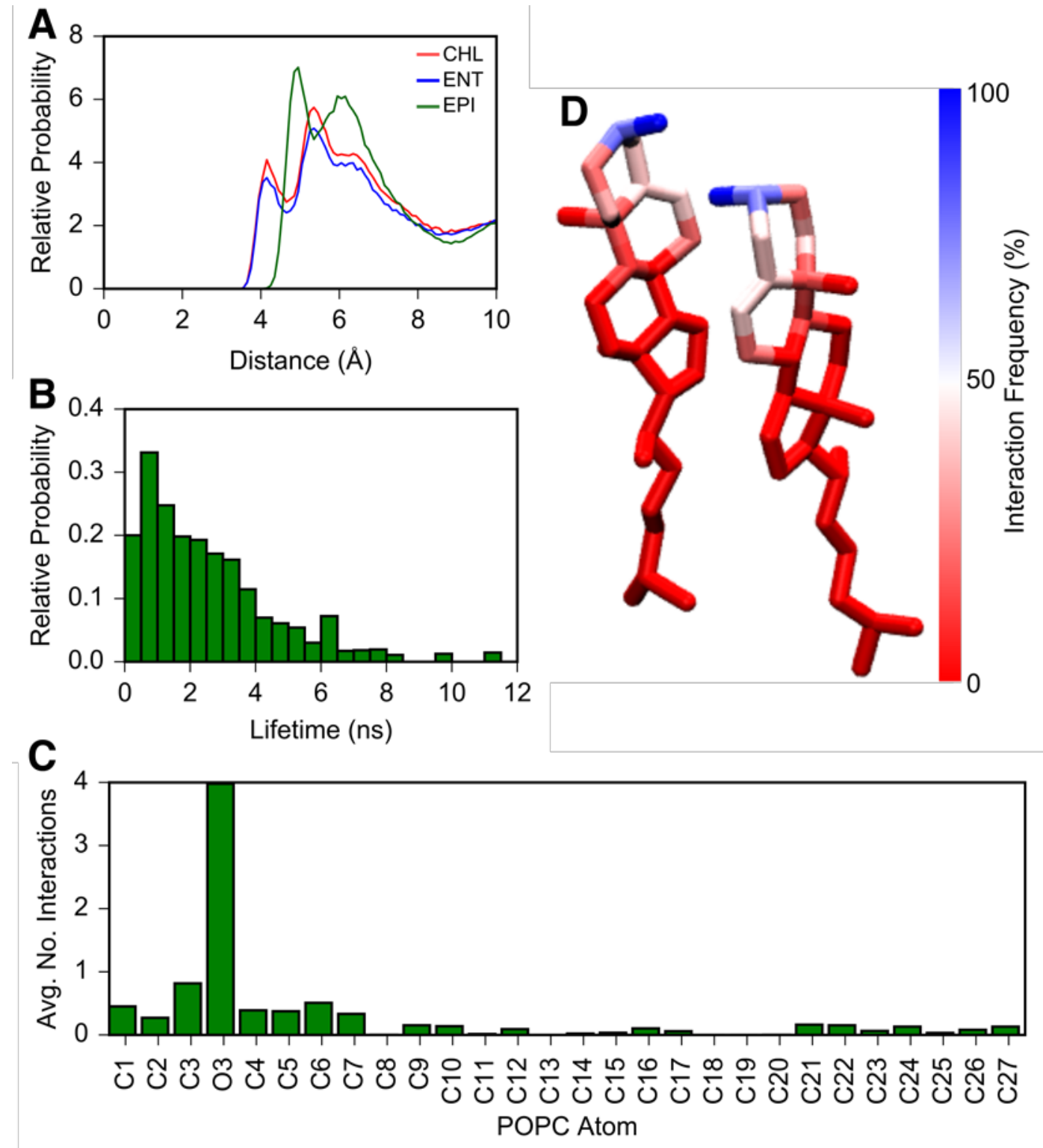

\section{DISCUSSION}


Of the cholesterol isomers in this study, nat-cholesterol exhibits the greatest influence on the physical properties of the membrane, almost exactly replicated by its enantiomer. Epi-cholesterol, on the other hand, exerts a weaker ordering effect as represented by the $S_{C D}$ order parameter, manifesting in a decrease in bilayer thickness. Such deviations can be attributed to adjustment of the position and tilt angle of epicholesterol in the phospholipid bilayer, and modification of the cholesterol surface available to interact with other cholesterol and phospholipid molecules.

The simulations presented here establish a direct link between the isomerisation of the cholesterol hydroxyl group and the occurrence of novel cholesterol oligomer conformations, specifically epi-cholesterol dimers stabilised by intermolecular $\mathrm{H}$ bonding. Despite significant interest in cholesterol aggregation and raft formation, experimental evidence concerning the existence of cholesterol dimers is negligible. Face-to-face dimers, stabilised by van der Waals interactions between the cholesterol $\alpha$-faces, were first proposed by Martin and Yeagle, ${ }^{52}$ and have been examined in detail in recent years by computational means. ${ }^{53}$ A study by Bandara et al, using unbiased MD simulations, calculated dimer lifetime at the order of hundreds of picoseconds, with instances of a nanosecond lifetime more prevalent at higher cholesterol and sphingomyelin concentrations. ${ }^{53}$ Furthermore, Andoh and coworkers used thermodynamic integration to calculate the free-energy profile of cholesterol dimerization. ${ }^{54}$ Cholesterol molecules separated by $~ 10-15 \AA$ and interspersed by POPC acyl tails displayed an attractive interaction, with a well depth of $-3.5 \mathrm{~kJ} \mathrm{~mol}^{-1}$. This value approximates to a thermal energy of $\sim 310 \mathrm{~K}$, highlighting the temperature dependence of the dimerization process. ${ }^{54}$ Additional computational studies have noted the instability of artificial aggregates that rapidly dissociate to approximately monomeric units, ${ }^{55}$ and the increased likelihood of cholesterol aggregates at elevated concentrations, ${ }^{56}$ as well as the direct observation of raft formation in both all-atom and coarse-grained MD simulations. ${ }^{22,57-59}$ In this case, it is apparent the H-bonding properties of epi-cholesterol significantly enhance the stability of the dimer, resulting in an average lifetime at least one order of magnitudes greater than those previously calculated. The increased frequency of such assemblies inadvertently reduces the availability of the cholesterol $\alpha$-face, which forms the dimer interface, and can therefore modify the molecular interaction between other cholesterol and phospholipid molecules, as well as membrane proteins. A growing number of studies predicate a direct relationship between membrane cholesterol and GPCR 
oligomerisation, ${ }^{60-62}$ with the involvement of the face-to-face dimer in the formation of a GPCR functional dimer postulated in earlier works. ${ }^{63}$ It is therefore conceivable that evolved epi-cholesterol dimer species may directly interact with membrane proteins, translating as the observed functional changes. In the 5- $\mathrm{HT}_{1 \mathrm{~A}}$ receptor, for example, the binding affinity of extracellular ligands is directly related to the presence of specific cholesterol isomers, nat- and ent- cholesterol in solubilized native hippocampal membranes. ${ }^{20}$ Specific interactions between cholesterol and the 5-HT $1 \mathrm{~A}$ receptor will be explored in future computational studies.

Bandari and coworkers have identified a close correlation between the activity of the $5-\mathrm{HT}_{1 \mathrm{~A}}$ receptor and the membrane dipole potential. ${ }^{17}$ Dipole potential has been implicated in the modulation of channel gating in voltage-gated ion channels, ${ }^{64}$ the formation of lipid rafts, ${ }^{65}$ the clustering and ligand binding affinity of receptor tyrosine kinases ${ }^{65}$ and the mechanism of anaesthetic action, and other small molecules. $^{66}$

The membrane dipole potential emanates from the ordered orientation of lipid headgroups and associated water dipoles in the polar region separating the hydrocarbon interior from the solvent. ${ }^{67}$ Regulation of membrane dipole potential by cholesterol has been ascribed to a number of factors, including (1) reorientation of the $\mathrm{P}^{+}-\mathrm{N}^{-}$dipole in the phospholipid headgroup towards the membrane plane, (2) membrane condensation, resulting in increased charge density, (3), an ordered water network in the interfacial region, and (4) the inherent dipole of cholesterol molecule itself. $^{14,64}$ These hypotheses will be discussed in relation to the known experimental trend for the bilayer compositions under investigation: epi-cholesterol $(\sim 338 \mathrm{mV})<$ POPC-only $(\sim 369 \mathrm{mV})<$ ent-cholesterol $(\sim 480 \mathrm{mV})<$ nat-cholesterol $(\sim 500 \mathrm{mV}) .{ }^{17}$ Immediately, it can be proposed that conformational changes in the phospholipid headgroup do not contribute to modification of the dipole potential in phospholipidcholesterol bilayers, as negligible differences are observed in the behaviour of the $\mathrm{P}^{+}$$\mathrm{N}^{-}$dipole. The orientation of the $\mathrm{P}^{+}-\mathrm{N}^{-}$dipole has been proposed as a significant contributor to the difference in dipole potential observed between PC and PE headgroups, as well as the dynamics of the interfacial water. ${ }^{68}$ The $\mathrm{PE} \mathrm{P}^{+}-\mathrm{N}^{-}$dipole is thought to occupy a position more parallel to the membrane, relative to PC, maintain optimal contacts with the phospholipid acyl chains. Concurrently, the negative contribution to the dipole potential is reduced, resulting in a more positive overall 
value. This theory is supported by quadrupole splitting of NMR spectra of deuterated phospholipids, but not applicable in the context of this study.

Evidence has been provided in this study that the inherent dipole moment of cholesterol provides a positive contribution to the membrane dipole potential. Notably, we demonstrate that the observed augmentation is dampened in the presence of epi-cholesterol, providing a less positive increase to the dipole potential. In a previous study examining cholesterol and four derivatives (6-ketocholestanol, 4cholesten-3-one, coprostanol, and 5-cholesten-3 $\beta$-ol-7-one), $10-30 \%$ of the total change in the dipole potential was attributed to the intrinsic dipole moments of the molecules. ${ }^{14}$ The authors credit the additional increase to membrane condensation and modifications in water penetration of the membrane. For the cholesterol isomers examined here, the calculated area per lipid is similar in the cholesterol isomers examined, indicating an equable increase in dipole potential as a result of higher packing. ${ }^{69}$ The relationship between headgroup hydration is more complex, however. The interaction of water with phospholipid bilayers has been investigated in detail in numerous studies; ${ }^{70-73}$ revealing water molecules specifically oriented parallel to the bilayer normal, with hydrogen atoms directed towards the membrane, thus providing positive contributions to the dipole potential. ${ }^{64}$ In theory, therefore, further hydration will increase the membrane dipole potential, whilst disruption will decrease it. Using MD simulations Villareal and coworkers attributed the observed changes in dipole potential of DMPC membranes in the presence of trehalose to replacement of lipidwater $\mathrm{H}$-bonds with lipid-trehalose $\mathrm{H}$-bonds, despite maintenance of the overall number of H-bonds. ${ }^{74}$ Overall, we observe the loss of a single H-bond between POPC molecules and water molecules when cholesterol isomers are included in the bilayer systems, with a further H-bond replaced with nat- and ent-cholesterol hydroxyl groups, and to a lesser extent with epi-cholesterol. We must also note the additional loss of H-bonding between epi-cholesterol and water, despite increased hydration, relative to nat-cholesterol and ent-cholesterol. As a consequence, it is apparent cholesterol isomers modulate the behaviour of interfacial water molecules in the presence of distinct cholesterol isomers, and hence the effective dielectric constant of the interfacial region. However, it is difficult to establish the exact contribution to the dipole potential, relative to the cholesterol-free bilayer.

Finally, differential ion binding to phospholipid headgroups is also exposed within this study, highlighting further divergence in the interfacial region. In general, 
physiologically relevant cations, $\mathrm{Na}^{+}, \mathrm{K}^{+}$and $\mathrm{Ca}^{2+}$ are known to bind to carbonyl and phosphate atoms in phospholipid molecules, whilst $\mathrm{Cl}^{-}$ions remain loosely associated to the bilayer surface, generating an effectively positive charged surface, and biasing other interactions in the interfacial region. ${ }^{75-77}$ Magarkar and colleagues uncovered an inverse correlation between cholesterol concentration and $\mathrm{Na}^{+}$binding, parallel to reductions in the positive surface potential, using a combination of computational and experimental means. ${ }^{78}$ Such changes were attributed to the replacement of high affinity phospholipid binding sites with low affinity cholesterol binding sites, preferred interactions between phospholipids and cholesterol and increased hydrophobicity of the membrane. This is supported by recent reports utilizing atomic force microscopy and Kelvin force microscopy indicating distinct regions of electric surface potential directly associated with cholesterol nanodomain formation. ${ }^{79}$ Further to this, data from Lairion and Disalvo indicates that the dipole and surface potentials of bilayers are interdependent. ${ }^{80}$ In this case, subsidence of cation $\left(\mathrm{K}^{+}\right)$binding is intensified when epi-cholesterol is present, signalling the greatest displacement of the membrane surface potential to less positive values, and imposing further variance to the membrane dipole potential.

Overall, interpretation of the data presented in this study reveals that the intricate balance between membrane condensation, the dipole moment of sterol molecules, and the behaviour of interfacial water molecules ions underlie the observed differences in the membrane dipole potential in cholesterol-containing bilayers. The influence of the $\mathrm{P}^{+}-\mathrm{N}^{-}$vector can be discounted in the context of this study.

\section{CONCLUSIONS}

Using extensive MD simulations, totalling $2 \mu \mathrm{s}$, the behaviour of epi-cholesterol in an unsaturated phospholipid bilayer has been characterised, and compared to natcholesterol and ent-cholesterol, as well as a cholesterol-free phospholipid bilayer. Slight differences in membrane thickness, area per lipid, interdigitation fraction and $\mathrm{S}_{\mathrm{CD}}$ order parameter are observed, relative to nat-cholesterol and ent-cholesterol which display identical properties throughout, in agreement with experimental studies. This can be attributed to minor deviations of the position and tilt angle of epicholesterol, as well as an observable population of long-lasting epi-cholesterol dimers, which are directly stabilised by H-bonding of the axial hydroxyl group on the cholesterol $\alpha$-face. 
Noteworthy variations are evident in the membrane-water interfacial region when epicholesterol is present, with the phospholipid SASA and interaction profile more closely resembling that of a cholesterol-free membrane. Following this, we have considered in detail how the divergent atomic-level interactions directly influence the dipole potential of the membrane, and provide a comprehensive mechanism for the action of cholesterol in this respect.

The membrane dipole potential is known to regulate transport processes, directly through the plasma membrane and also via embedded proteins. Consequently, understanding how the membrane dipole potential is modulated by various factors, such as the inclusion of small molecules, is of intense interest in the pharmaceutical industry. Therefore, it is desired that the outcomes of this study provide crucial insights in this area, and contribute to our current understanding of the behaviour and role of the cholesterol in the cell membrane.

Acknowledgments. This work was supported by the Biotechnology and Biological Sciences Research Council (BB/L015269/1) and Pfizer Neusentis via a studentship to V. Oakes. This project was possible thanks to a computational resource allocation from (i) RES in Magerit based in Universidad Autonoma de Madrid (Spain) and from (ii) DECI in Archer based in EPCC (UK), Marconi based in CINECA (Italy), and SuperMUC based in Juelich (Germany) with support from PRACE.

\section{REFERENCES}

1. Ikonen, E., Cellular cholesterol trafficking and compartmentalization. Nature reviews. Molecular cell biology 2008, 9 (2), 125-38.

2. Leathes, J. B., Croonian Lectures ON THE ROLE OF FATS IN VITAL PHENOMENA. The Lancet 205 (5304), 853-856.

3. McConnell, H. M.; Radhakrishnan, A., Condensed complexes of cholesterol and phospholipids. Biochimica et biophysica acta 2003, 1610 (2), 159-73.

4. Westover, E. J.; Covey, D. F.; Brockman, H. L.; Brown, R. E.; Pike, L. J., Cholesterol depletion results in site-specific increases in epidermal growth factor receptor phosphorylation due to membrane level effects. Studies with cholesterol enantiomers. The Journal of biological chemistry 2003, 278 (51), 51125-33.

5. Westover, E. J.; Covey, D. F., The enantiomer of cholesterol. The Journal of membrane biology 2004, 202 (2), 61-72.

6. Covey, D. F., ent-Steroids: novel tools for studies of signaling pathways. Steroids 2009, 74 (7), 577-85.

7. Mannock, D. A.; McIntosh, T. J.; Jiang, X.; Covey, D. F.; McElhaney, R. N., Effects of natural and enantiomeric cholesterol on the thermotropic phase behavior and structure of egg sphingomyelin bilayer membranes. Biophysical journal 2003, 84 (2 Pt 1), 1038-46.

8. Demel, R. A.; Bruckdorfer, K. R.; van Deenen, L. L. M., Structural requirements of sterols for the interaction with lecithin at the air-water interface. Biochimica et Biophysica Acta (BBA) - Biomembranes 1972, 255 (1), 311-320. 
9. Dufourc, E. J.; Parish, E. J.; Chitrakorn, S.; Smith, I. C. P., Structural and dynamical details of cholesterol-lipid interaction as revealed by deuterium NMR. Biochemistry 1984, 23 (25), 6062-6071.

10. Murari, R.; Murari, M. P.; Baumann, W. J., Sterol orientations in phosphatidylcholine liposomes as determined by deuterium NMR. Biochemistry 1986, 25 (5), 1062-1067.

11. Cheetham, J. J.; Wachtel, E.; Bach, D.; Epand, R. M., Role of the stereochemistry of the hydroxyl group of cholesterol and the formation of nonbilayer structures in phosphatidylethanolamines. Biochemistry 1989, 28 (22), 8928-8934.

12. Liu, Y.; Chipot, C.; Shao, X.; Cai, W., The effects of 7-dehydrocholesterol on the structural properties of membranes. Physical biology 2011, 8 (5), 056005.

13. Huster, D.; Scheidt, H. A.; Arnold, K.; Herrmann, A.; Müller, P., Desmosterol May Replace Cholesterol in Lipid Membranes. Biophysical journal 2005, 88 (3), 1838-1844.

14. Starke-Peterkovic, T.; Turner, N.; Vitha, M. F.; Waller, M. P.; Hibbs, D. E.; Clarke, R. J., Cholesterol Effect on the Dipole Potential of Lipid Membranes. Biophysical journal 2006, 90 (11), 4060-4070.

15. Haldar, S.; Kanaparthi, R. K.; Samanta, A.; Chattopadhyay, A., Differential effect of cholesterol and its biosynthetic precursors on membrane dipole potential. Biophysical journal 2012, 102 (7), 1561-9.

16. Singh, P.; Haldar, S.; Chattopadhyay, A., Differential effect of sterols on dipole potential in hippocampal membranes: Implications for receptor function. Biochimica et Biophysica Acta (BBA) - Biomembranes 2013, 1828 (3), 917-923. 17. Bandari, S.; Chakraborty, H.; Covey, D. F.; Chattopadhyay, A., Membrane dipole potential is sensitive to cholesterol stereospecificity: Implications for receptor function. Chemistry and Physics of Lipids 2014, 184, 25-29.

18. D'Avanzo, N.; Hyrc, K.; Enkvetchakul, D.; Covey, D. F.; Nichols, C. G., Enantioselective Protein-Sterol Interactions Mediate Regulation of Both Prokaryotic and Eukaryotic Inward Rectifier K+ Channels by Cholesterol. PLOS ONE 2011, 6 (4), e19393.

19. Sooksawate, T.; Simmonds, M. A., Influence of membrane cholesterol on modulation of the $\mathrm{GABA}(\mathrm{A})$ receptor by neuroactive steroids and other potentiators. British Journal of Pharmacology 2001, 134 (6), 1303-1311.

20. Jafurulla, M.; Rao, B. D.; Sreedevi, S.; Ruysschaert, J. M.; Covey, D. F.; Chattopadhyay, A., Stereospecific requirement of cholesterol in the function of the serotonin1A receptor. Biochim Biophys Acta 2014, 1838 (1 Pt B), 158-63.

21. Kristiana, I.; Luu, W.; Stevenson, J.; Cartland, S.; Jessup, W.; Belani, J. D.; Rychnovsky, S. D.; Brown, A. J., Cholesterol through the looking glass: ability of its enantiomer to also elicit homeostatic responses. Journal of Biological Chemistry 2012.

22. Berkowitz, M. L., Detailed molecular dynamics simulations of model biological membranes containing cholesterol. Biochimica et Biophysica Acta (BBA) Biomembranes 2009, 1788 (1), 86-96.

23. Sengupta, D.; Chattopadhyay, A., Molecular dynamics simulations of GPCRcholesterol interaction: An emerging paradigm. Biochim Biophys Acta 2015, 1848 (9), 1775-82.

24. Grouleff, J.; Irudayam, S. J.; Skeby, K. K.; Schiott, B., The influence of cholesterol on membrane protein structure, function, and dynamics studied by molecular dynamics simulations. Biochim Biophys Acta 2015.

25. Róg, T.; Pasenkiewicz-Gierula, M., Effects of Epicholesterol on the Phosphatidylcholine Bilayer: A Molecular Simulation Study. Biophys. J. 2003, 84 (3), 1818-1826.

26. Jo, S.; Kim, T.; Iyer, V. G.; Im, W., CHARMM-GUI: a web-based graphical user interface for CHARMM. Journal of computational chemistry 2008, 29 (11), 185965. 
27. Jo, S.; Lim, J. B.; Klauda, J. B.; Im, W., CHARMM-GUI Membrane Builder for Mixed Bilayers and Its Application to Yeast Membranes. Biophysical Journal 2009, 97 (1), 50-58.

28. Wu, E. L.; Cheng, X.; Jo, S.; Rui, H.; Song, K. C.; Dávila - Contreras, E. M.; Qi, Y.; Lee, J.; Monje - Galvan, V.; Venable, R. M., CHARMM - GUI Membrane Builder toward realistic biological membrane simulations. Journal of computational chemistry 2014, 35 (27), 1997-2004.

29. Humphrey, W.; Dalke, A.; Schulten, K., VMD: Visual molecular dynamics. Journal of Molecular Graphics 1996, 14 (1), 33-38.

30. Phillips, J. C.; Braun, R.; Wang, W.; Gumbart, J.; Tajkhorshid, E.; Villa, E.; Chipot, C.; Skeel, R. D.; Kale, L.; Schulten, K., Scalable molecular dynamics with NAMD. Journal of computational chemistry 2005, 26 (16), 1781-802.

31. Klauda, J. B.; Venable, R. M.; Freites, J. A.; O'Connor, J. W.; Tobias, D. J.; Mondragon-Ramirez, C.; Vorobyov, I.; MacKerell Jr, A. D.; Pastor, R. W., Update of the CHARMM all-atom additive force field for lipids: validation on six lipid types. The journal of physical chemistry B 2010, 114 (23), 7830-7843.

32. Noskov, S. Y.; Berneche, S.; Roux, B., Control of ion selectivity in potassium channels by electrostatic and dynamic properties of carbonyl ligands. Nature 2004, 431 (7010), 830-834.

33. Jorgensen, W. L.; Chandrasekhar, J.; Madura, J. D.; Impey, R. W.; Klein, M. L., Comparison of simple potential functions for simulating liquid water. The Journal of chemical physics 1983, 79 (2), 926-935.

34. Lim, J. B.; Rogaski, B.; Klauda, J. B., Update of the Cholesterol Force Field Parameters in CHARMM. The Journal of Physical Chemistry B 2012, 116 (1), 203210.

35. Darden, T.; York, D.; Pedersen, L., Particle mesh Ewald: An N· $\log (\mathrm{N})$ method for Ewald sums in large systems. The Journal of Chemical Physics 1993, 98 (12), 10089-10092.

36. Verlet, L., Computer" experiments" on classical fluids. I. Thermodynamical properties of Lennard-Jones molecules. Physical review 1967, 159 (1), 98.

37. Miyamoto, S.; Kollman, P. A., SETTLE: an analytical version of the SHAKE and RATTLE algorithm for rigid water models. J. Comput. Chem. 1992, 13 (8), 952962.

38. Feller, S. E.; Zhang, Y.; Pastor, R. W.; Brooks, B. R., Constant pressure molecular dynamics simulation: the Langevin piston method. The Journal of chemical physics 1995, 103 (11), 4613-4621.

39. Martyna, G. J.; Tobias, D. J.; Klein, M. L., Constant pressure molecular dynamics algorithms. The Journal of Chemical Physics 1994, 101 (5), 4177-4189.

40. Guixa-Gonzalez, R.; Rodriguez-Espigares, I.; Ramirez-Anguita, J. M.; CarrioGaspar, P.; Martinez-Seara, H.; Giorgino, T.; Selent, J., MEMBPLUGIN: studying membrane complexity in VMD. Bioinformatics (Oxford, England) 2014, 30 (10), 147880.

41. Vermeer, L. S.; de Groot, B. L.; Reat, V.; Milon, A.; Czaplicki, J., Acyl chain order parameter profiles in phospholipid bilayers: computation from molecular dynamics simulations and comparison with $2 \mathrm{H}$ NMR experiments. European biophysics journal : EBJ 2007, 36 (8), 919-31.

42. Giorgino, T., Computing 1-D atomic densities in macromolecular simulations: The density profile tool for VMD. Computer Physics Communications 2014, 185 (1), 317-322.

43. Aksimentiev, A.; Schulten, K., Imaging $<$ em $>\& \# \times 3 b 1 ;</ e m>-H e m o l y s i n$ with Molecular Dynamics: Ionic Conductance, Osmotic Permeability, and the Electrostatic Potential Map. Biophysical journal 88 (6), 3745-3761. 
44. Kučerka, N.; Nieh, M.-P.; Katsaras, J., Fluid phase lipid areas and bilayer thicknesses of commonly used phosphatidylcholines as a function of temperature. Biochimica et Biophysica Acta (BBA) - Biomembranes 2011, 1808 (11), 2761-2771. 45. Olsen, B. N.; Schlesinger, P. H.; Ory, D. S.; Baker, N. A., 25Hydroxycholesterol Increases the Availability of Cholesterol in Phospholipid Membranes. Biophysical journal 2011, 100 (4), 948-956.

46. Aittoniemi, J.; Róg, T.; Niemelä, P.; Pasenkiewicz-Gierula, M.; Karttunen, M.; Vattulainen, I., Tilt: Major Factor in Sterols' Ordering Capability in Membranes. The Journal of Physical Chemistry B 2006, 110 (51), 25562-25564.

47. Oldfield, E.; Meadows, M.; Rice, D.; Jacobs, R., Spectroscopic studies of specifically deuterium labeled membrane systems. Nuclear magnetic resonance investigation of the effects of cholesterol in model systems. Biochemistry 1978, 17 (14), 2727-2740.

48. Urbina, J. A.; Pekerar, S.; Le, H.-b.; Patterson, J.; Montez, B.; Oldfield, E., Molecular order and dynamics of phosphatidylcholine bilayer membranes in the presence of cholesterol, ergosterol and lanosterol: a comparative study using $2 \mathrm{H}-$, 13C- and 31P-NMR spectroscopy. Biochimica et Biophysica Acta (BBA) Biomembranes 1995, 1238 (2), 163-176.

49. Jacobs, R.; Oldfield, E., Deuterium nuclear magnetic resonance investigation of dimyristoyllecithin--dipalmitoyllecithin and dimyristoyllecithin--cholesterol mixtures. Biochemistry 1979, 18 (15), 3280-5.

50. Róg, T.; Pasenkiewicz-Gierula, M.; Vattulainen, I.; Karttunen, M., Ordering effects of cholesterol and its analogues. Biochimica et Biophysica Acta (BBA) Biomembranes 2009, 1788 (1), 97-121.

51. Kulig, W.; Tynkkynen, J.; Javanainen, M.; Manna, M.; Rog, T.; Vattulainen, I.; Jungwirth, P., How well does cholesteryl hemisuccinate mimic cholesterol in saturated phospholipid bilayers? Journal of molecular modeling 2014, 20 (2), 2121.

52. Martin, R. B.; Yeagle, P. L., Models for lipid organization in cholesterolphospholipid bilayers including cholesterol dimer formation. Lipids 1978, 13 (9), 5947.

53. Bandara, A.; Panahi, A.; Pantelopulos, G. A.; Straub, J. E., Exploring the structure and stability of cholesterol dimer formation in multicomponent lipid bilayers. Journal of computational chemistry 2017, 38 (16), 1479-1488.

54. Andoh, Y.; Oono, K.; Okazaki, S.; Hatta, I., A molecular dynamics study of the lateral free energy profile of a pair of cholesterol molecules as a function of their distance in phospholipid bilayers. The Journal of Chemical Physics 2012, 136 (15), 155104.

55. Dai, J.; Alwarawrah, M.; Huang, J., Instability Of Cholesterol Clusters In Lipid Bilayers And The Cholesterol's Umbrella Effect. The journal of physical chemistry. $B$ 2010, 114 (2), 840.

56. O'Connor, J. W.; Klauda, J. B., Lipid Membranes with a Majority of Cholesterol: Applications to the Ocular Lens and Aquaporin 0. The Journal of Physical Chemistry B 2011, 115 (20), 6455-6464.

57. Pandit, S. A.; Bostick, D.; Berkowitz, M. L., Complexation of Phosphatidylcholine Lipids with Cholesterol. Biophysical journal 2004, 86 (3), 13451356.

58. Marrink, S. J.; de Vries, A. H.; Tieleman, D. P., Lipids on the move: Simulations of membrane pores, domains, stalks and curves. Biochimica et Biophysica Acta (BBA) - Biomembranes 2009, 1788 (1), 149-168.

59. Davis, R. S.; Sunil Kumar, P. B.; Sperotto, M. M.; Laradji, M., Predictions of Phase Separation in Three-Component Lipid Membranes by the MARTINI Force Field. The Journal of Physical Chemistry B 2013, 117 (15), 4072-4080.

60. Gahbauer, S.; Böckmann, R. A., Membrane-Mediated Oligomerization of G Protein Coupled Receptors and Its Implications for GPCR Function. Frontiers in Physiology 2016, 7, 494. 
61. Prasanna, X.; Chattopadhyay, A.; Sengupta, D., Cholesterol Modulates the Dimer Interface of the $\beta(2)$-Adrenergic Receptor via Cholesterol Occupancy Sites. Biophys. J. 2014, 106 (6), 1290-1300.

62. Prasanna, X.; Sengupta, D.; Chattopadhyay, A., Cholesterol-dependent Conformational Plasticity in GPCR Dimers. 2016, 6, 31858.

63. Fantini, J.; Barrantes, F. J., How cholesterol interacts with membrane proteins: an exploration of cholesterol-binding sites including CRAC, CARC, and tilted domains. Frontiers in physiology 2013, 4, 31.

64. Pearlstein, R. A.; Dickson, C. J.; Hornak, V., Contributions of the membrane dipole potential to the function of voltage-gated cation channels and modulation by small molecule potentiators. Biochimica et Biophysica Acta (BBA) - Biomembranes 2017, 1859 (2), 177-194.

65. Kovács, T.; Batta, G.; Zákány, F.; Szöllösi, J.; Nagy, P., The dipole potential correlates with lipid raft markers in the plasma membrane of living cells. Journal of Lipid Research 2017.

66. Davis, B. M.; Brenton, J.; Davis, S.; Shamsher, E.; Sisa, C.; Grgic, L.; Cordeiro, M. F., Modulation of the membrane dipole potential: a prospective mechanism to differentiate anaesthetic/non-anaesthetic activity and a tool to assess susceptibility. Journal of Lipid Research 2017.

67. Gawrisch, K.; Ruston, D.; Zimmerberg, J.; Parsegian, V. A.; Rand, R. P.; Fuller, N., Membrane dipole potentials, hydration forces, and the ordering of water at membrane surfaces. Biophysical journal 1992, 61 (5), 1213-1223.

68. Starke-Peterkovic, T.; Clarke, R. J., Effect of headgroup on the dipole potential of phospholipid vesicles. European Biophysics Journal 2009, 39 (1), 103. 69. Clarke, R. J., Effect of lipid structure on the dipole potential of phosphatidylcholine bilayers. Biochimica et Biophysica Acta (BBA) - Biomembranes 1997, 1327 (2), 269-278.

70. Re, S.; Nishima, W.; Tahara, T.; Sugita, Y., Mosaic of Water Orientation Structures at a Neutral Zwitterionic Lipid/Water Interface Revealed by Molecular Dynamics Simulations. The Journal of Physical Chemistry Letters 2014, 5 (24), 43434348.

71. Abd Halim, K. B.; Koldso, H.; Sansom, M. S. P., Interactions of the EGFR juxtamembrane domain with PIP2-containing lipid bilayers: Insights from multiscale molecular dynamics simulations. Biochimica et biophysica acta 2015, 1850 (5), 101725.

72. Krylov, N. A.; Pentkovsky, V. M.; Efremov, R. G., Nontrivial Behavior of Water in the Vicinity and Inside Lipid Bilayers As Probed by Molecular Dynamics Simulations. ACS Nano 2013, 7 (10), 9428-9442.

73. Davis, J. E.; Rahaman, O.; Patel, S., Molecular Dynamics Simulations of a DMPC Bilayer Using Nonadditive Interaction Models. Biophysical journal 96 (2), 385402.

74. Villarreal, M. A.; Díaz, S. B.; Disalvo, E. A.; Montich, G. G., Molecular Dynamics Simulation Study of the Interaction of Trehalose with Lipid Membranes. Langmuir 2004, 20 (18), 7844-7851.

75. Berkowitz, M. L.; Bostick, D. L.; Pandit, S., Aqueous Solutions next to Phospholipid Membrane Surfaces: Insights from Simulations. Chemical Reviews 2006, 106 (4), 1527-1539.

76. Stępniewski, M.; Bunker, A.; Pasenkiewicz-Gierula, M.; Karttunen, M.; Róg, T., Effects of the Lipid Bilayer Phase State on the Water Membrane Interface. The Journal of Physical Chemistry B 2010, 114 (36), 11784-11792.

77. Magarkar, A.; Karakas, E.; Stepniewski, M.; Róg, T.; Bunker, A., Molecular Dynamics Simulation of PEGylated Bilayer Interacting with Salt lons: A Model of the Liposome Surface in the Bloodstream. The Journal of Physical Chemistry B 2012, 116 (14), 4212-4219. 
78. Magarkar, A.; Dhawan, V.; Kallinteri, P.; Viitala, T.; Elmowafy, M.; Róg, T.; Bunker, A., Cholesterol level affects surface charge of lipid membranes in saline solution. Scientific Reports 2014, 4, 5005.

79. Drolle, E.; Bennett, W. F. D.; Hammond, K.; Lyman, E.; Karttunen, M.; Leonenko, Z., Molecular dynamics simulations and Kelvin probe force microscopy to study of cholesterol-induced electrostatic nanodomains in complex lipid mixtures. Soft Matter 2017, 13 (2), 355-362.

80. Lairion, F.; Disalvo, E. A., Effect of Dipole Potential Variations on the Surface Charge Potential of Lipid Membranes. The Journal of Physical Chemistry B 2009, 113 (6), 1607-1614. 